\title{
The LLAMA Middleware Support for Accountable Service-Oriented Architecture
}

\author{
Mark Panahi, Kwei-Jay Lin, Yue Zhang, Soo-Ho Chang, Jing Zhang, \\ and Leonardo Varela
}

\author{
Department of Electrical Engineering and Computer Science \\ University of California, Irvine \\ Irvine, CA 92697, USA
}

\begin{abstract}
Enterprises are turning to SOA for the flexible deployment of business processes. While current monitoring tools can detect service execution problems in an enterprise's servers and report such problems to human decision makers, they may not closely monitor the external services they use, diagnose the root cause of process problems, and automatically reconfigure the process to replace faulty services. This paper presents the LLAMA middleware support for service process monitoring, run-time management, and configuration. Instances of accountability agents are deployed to selectively monitor some services' performance. These agents in turn allow LLAMA's Accountability Authority (AA) to diagnose process problems and apply any necessary reconfiguration. The project also builds tools to simplify the setup and deployment of LLAMA components. Our experimental results indicate that using LLAMA contributes only a modest amount of system overhead, and that the diagnosis process is swift and sufficiently accurate.
\end{abstract}

\section{Introduction}

Service-oriented architecture (SOA) is a software architecture for composing loosely-coupled services into one cohesive business process (BP) [12 3]. Enterprises can benefit from SOA to dynamically create BP's by integrating both legacy and new services. Some BP's may involve external services supplied by third-party partners/providers and invoke them with a service-level agreement (SLA) on the QoS performance of those services.

Service providers must be held accountable for the failures in the services they offer. However, identifying the source of SLA violations or service failures in a $\mathrm{BP}$ can be difficult. BP's can be very complicated, with many execution branches and involving services from several different providers. Performances from services may have dependencies; an irregular performance from one service may cause another to fail unexpectedly. In order to investigate such problems, the behavior of services that belong to a BP must be continuously monitored, logged, aggregated, and analyzed 41. Harnessing such vital information requires intelligent support from the service infrastructure.

A. Bouguettaya, I. Krueger, and T. Margaria (Eds.): ICSOC 2008, LNCS 5364, pp. $180-1942008$.

(C) Springer-Verlag Berlin Heidelberg 2008 
Given the dynamic and complex nature of many BP's, a powerful yet efficient mechanism is required to identify the source of problems when a BP has not delivered the service performance as expected. Such an error identification mechanism must be powerful enough to handle the complex and causal nature of service interactions and efficient enough to cope with a large number of service nodes without imposing significant system overhead.

In this paper, we present a middleware framework, called the inteLLigent Accountability Middleware Architecture (LLAMA), for accountable service-oriented computing. LLAMA includes three main components. The Accountability Service Bus (ASB) transparently and selectively monitors and records services, hosts (e.g. $\mathrm{CPU}$ ), and network behavior information for services running on it. LLAMA also deploys instances of monitoring Agents to observe and to inspect groups of services. Finally, an Accountability Authority (AA) is in charge of diagnosing performance problems in processes and applying desirable reconfiguration measures. The LLAMA project also provides useful tools to simplify the setup, selection and deployment of LLAMA components, as well as a friendly user interface to inspect the diagnosis status of all processes.

The contribution of our research is that we have implemented LLAMA as an intelligent SOA middleware framework to detect, diagnose and defuse QoS issues in BP's. Our project goes beyond current business activity monitoring (BAM) tools to add comprehensive error identification capabilities. Some of the unique features in LLAMA include:

1. efficient decisions to select only a subset of monitoring locations (called Evidence Channels) in a BP to reduce monitoring cost and overhead 4;

2. a powerful diagnosis engine (using the Baysian network model [5]) which conducts probabilistic analysis to identify the most probable service failures [4;

3. optimal Agent selection coupled with service selection so as to build BP's with a low overall cost including services and diagnosis [6];

4. intelligent capabilities for Agents to inspect individual service logs to confirm if a service is indeed faulty, and if so, whether the cause was due to service logic, host, or network behavior.

Following the work reported in [46], this paper concentrates on the LLAMA architecture, its components, implementation and performance study. We measure the extent to which monitoring and inspection add overhead to system performance. We also investigate how the accuracy of diagnosis varies according to the amount of time and resources devoted to the diagnostic process. Our experimental results indicate that using LLAMA contributes only a very modest amount of monitoring overhead, and the diagnosis process is swift and sufficiently accurate given reasonable time allowed.

The paper is organized as follows. Sec. 2 reviews the concept of accountability and challenges for building accountable SOA. Sec. 3 presents the LLAMA accountable middleware architecture. We present a performance study of the LLAMA implementation in Sec. 4. Related work is compared in Sec. 5. 


\section{Background}

\subsection{Challenges of Accountability in SOA}

As defined in the Merriam-Webster dictionary, accountability is "an obligation or willingness to accept responsibility or to account for one's actions". We apply the concept of accountability to SOA so that all services are regulated for effective QoS delivered to a BP, and all root causes of faulty service executions can be clearly identified, inspected, and defused to control damage. In our work, QoS parameters can include any attribute that can be measured and quantified, such as timeliness, throughput, reliability, and availability.

To achieve accountability in SOA, we have identified the following challenges introduced by SOA's inborn characteristics: 1) An SOA accountability mechanism should be capable of dealing with the causal relationship existing in service interactions and find the root cause of a process problem. 2) Probabilistic and statistical theory should be used to model the uncertainty inherent in networkbased workflows and server workloads. 3) The problem diagnosis mechanism needs to scale well in large-scale distributed SOA systems. 4) To prevent excessive overhead, the amount of service performance data collected should be as little as possible but still enough for a correct diagnosis to be made. 5) The trustworthiness of services needs to be continually evaluated based on accumulated service behavior data. All these important challenges have motivated the design and development of our accountability model, algorithms, and architecture. These issues have also been elaborated in 4 .

There are different degrees to which SOA systems can achieve these goals. At the very core, SOA systems must provide efficient monitoring feedback and error checking facilities on deployed services. Next, accountability systems may provide an intelligent and powerful diagnosis engine to analyze monitored data from the execution history and process structure. Finally, a mature accountability system must provide a means to reconfigure faulty processes and to prevent future problems. Depending on the application needs, the diagnosis and reconfiguration mechanism can be performed either offline or online, with the online approach more preferred so as to promptly react to problems as they occur.

\subsection{Current Monitoring Support in SOA}

The enterprise service bus (ESB) is a common service integration and deployment technology for SOA [7] that has been extended to provide support for monitoring and logging. Tools such as business activity monitoring (BAM) provide both analysis and visualization of data collected by ESB for the various services deployed on it. Users of BAM tools can view the performance of business processes and be alerted when problems occur. For example, a BAM tool may answer the question: "Why is it taking an average of 10 minutes for a representative to answer a customer's call?" The Cape Clear BAM $[\underline{8}$ is a commercial solution that claims to "combine an ESB with BAM to provide greater flexibility and ease-of-deployment within complex heterogeneous environments." Another example is the Saturn product working with the Mule ESB [9]. 
While current solutions to business process monitoring and problem diagnosis exist, they still have limitations. Current BAM tools usually report information via a dashboard or email alerts to human decision makers, who then manually initiate diagnosis and corrective actions. We foresee a future where enterprises will be integrating systems involving third-party, geographically dispersed services and components, and may wish to compose services dynamically and automatically. The LLAMA framework is therefore designed to perform automated diagnosis and reconfiguration based on either pre-specified or dynamically calculated service causal relationships.

\subsection{Transparency and Service Provider Participation in an Accountability Framework}

The LLAMA framework is designed to help enterprises with pinpointing responsible parties when a process execution has problems. To achieve this, transparency on the (internal) state of a service provider is critical to the success of the diagnosis. However, some service providers may not be willing to grant this transparency to external users. Service providers therefore need to consider the tradeoffs between transparency on one hand, and privacy and security on the other. Providers of "healthy" services actually will benefit by allowing its performance data to be reported in order to clear any fault responsibility. In other words, we believe transparency may be more valuable than privacy to most service providers.

In order to participate in the accountability framework, external service providers must install the LLAMA ASB (see Sec. 3.2) to produce an audit trail of their services, and to allow ASB to push performance data to Agents. In LLAMA, to give more flexibility, we design Agents to be accountability-related services that can be deployed by service clients, service providers, or any third party providers. The only requirement is that Agents can be selectively requested by the Accountability Authority to report data about services that belong to a specific process.

In the case that some service providers are not willing to release their performance data unconditionally, LLAMA allows service providers to choose among various levels of transparency. Simple Auditing requires the service provider to only install the ASB layer for their services. This activates data collection for such services. However, this data is stored within the server and only provided to the AA, via an assigned Agent, when diagnosis is required. Dynamic Monitoring requires the ASB installation to allow dynamic monitoring of services via Agents installed by the service provider. Agents deployed need only conform to a standard interface. Therefore, the advantage of this transparency level is that service providers may use their own Agents to participate in the diagnosis process. Dynamic Third-party Monitoring is similar to the previous level except that data is collected and processed by third-party "collateral" Agents.

For the rest of this paper, we assume all external services are running on an ASB instance and monitored by Agents deployed by LLAMA. This assumption simplifies our discussion. Support for other transparency levels will be addressed in the future. 


\section{The LLAMA Accountability Middleware Architecture}

Using SOA, the choices of which service to invoke at a specific instance may be made continuously depending on current service performance, cost, and many other factors. For a highly dynamic environment, few frameworks in existence are built to automate the analysis and identification of business process problems, and perform reconfigurations. In this section, we highlight the various components and features of the LLAMA framework that make automated process monitoring, analysis, and detection possible.

\subsection{LLAMA Overview}

Figure 1 shows an example of service system deployed on LLAMA. As discussed in Sec. 2.3, all service nodes are assumed to be deployed on the ASB. In addition to the service requester and services deployed, the Accountability Authority (AA) and Agents are the two main LLAMA components to be discussed in detail in the next section.

Agents are deployed on servers and selected by AA to monitor a BP. AA and Agents collaborate to perform run-time process monitoring, root-cause diagnosis, service network recovery, and service network optimization. Multiple Agents are selected by AA to address scalability requirements. Each Agent is put in charge of monitoring a subset of services (as depicted by the circles in Fig. 1) during the execution of the service process. When undesirable process outcomes are reported by monitors, Agents provide AA relevant service status information that serves as evidence for AA to diagnose the root causes of run-time process problems.

The Accountability Console provides a Web-based graphical user interface for LLAMA. The operations in the console are classified into three types of behaviors: 1) registering a business process and SLA requirements in AA, 2)

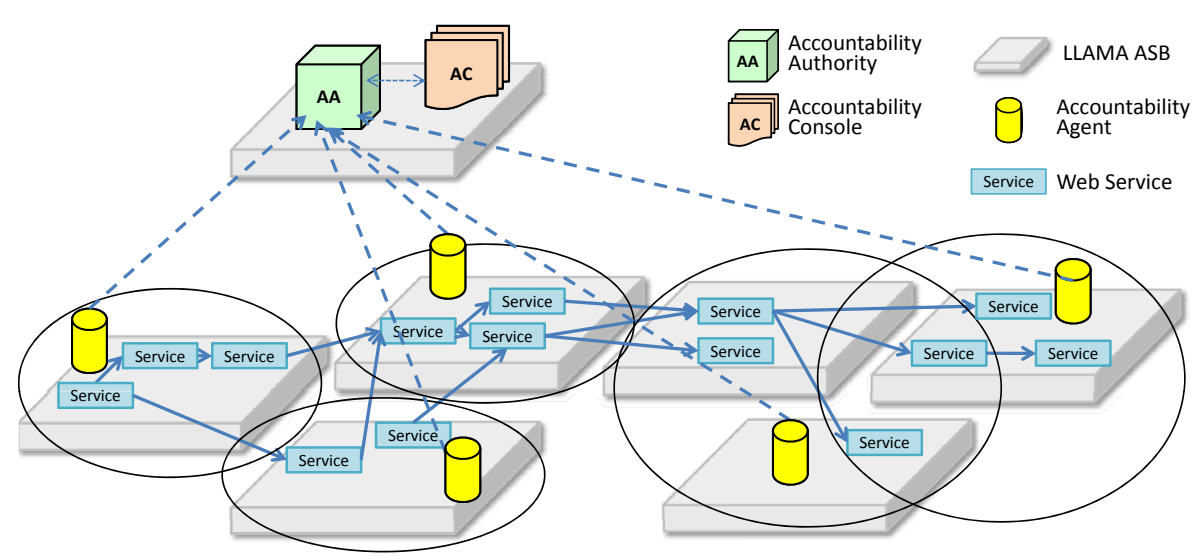

Fig. 1. An example layout of accountability framework 


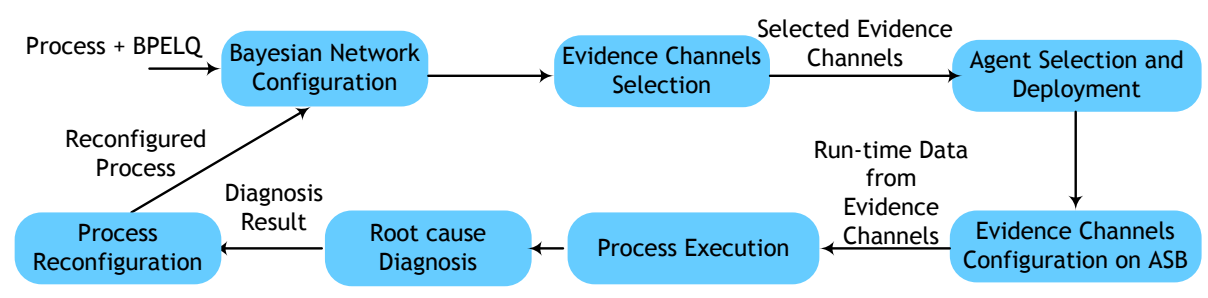

Fig. 2. Accountable SOA configuration and deployment flow

configuring AA parameters, and 3) reporting diagnosis results to users. The console provides the interface between human business process managers and the accountability framework through WSDM interfaces [10].

In addition to these components, LLAMA also has a QoS broker 11] (not in figure) which offers process composition and QoS-based service selection, in order to assist the service user in fulfilling user-defined end-to-end QoS requirements for a business process 12. QoS broker composes BPEL processes and defines the QoS constraint for each individual service in a process. Furthermore, LLAMA deploys trust and reputation brokers for evaluating, aggregating, and managing the reputation of services. A service's reputation is considered as a QoS parameter that affects service network composition: services with a better reputation are more likely to be invoked by users.

Figure 2 shows the steps of the accountable SOA configuration and deployment. Given a user's service request with a user-specified end-to-end QoS requirement, the QoS Broker first composes the business process to be executed. The QoS Broker selects individual services that make up the service process, and identify the QoS constraint for each individual service in the process. These are specified in a special language designed in our project, called BPELQ (for "BPEL with QoS"), and sent to AA. In AA, the Bayesian Network for this process is configured based on the process graph, as well as both historical and expected service performance data [4]. AA then runs the Evidence Channel Selection algorithm using information from the Bayesian analysis to yield the best locations for collecting data about the process. The Agents that can best cover the services in this process are also selected and deployed by AA [6]. In addition, the hosts of the selected evidence channels are configured on the ASBs, ready to send monitored data at regular intervals to respective Agents.

Once the process starts to execute, performance data about services and the process will be collected by the Evidence Channels and delivered to Agents. If any abnormal situation is detected by an Agent, it will inform AA to trigger Root Cause Diagnosis. AA will produce the list of the most likely faulty services (with their respective probability readings), instruct the Agents to confirm the service status for fault identification, and initiate a Process Reconfiguration to resolve the problem. In this way, the framework can detect problems when they occur, find the root cause, and select a reconfiguration plan. 


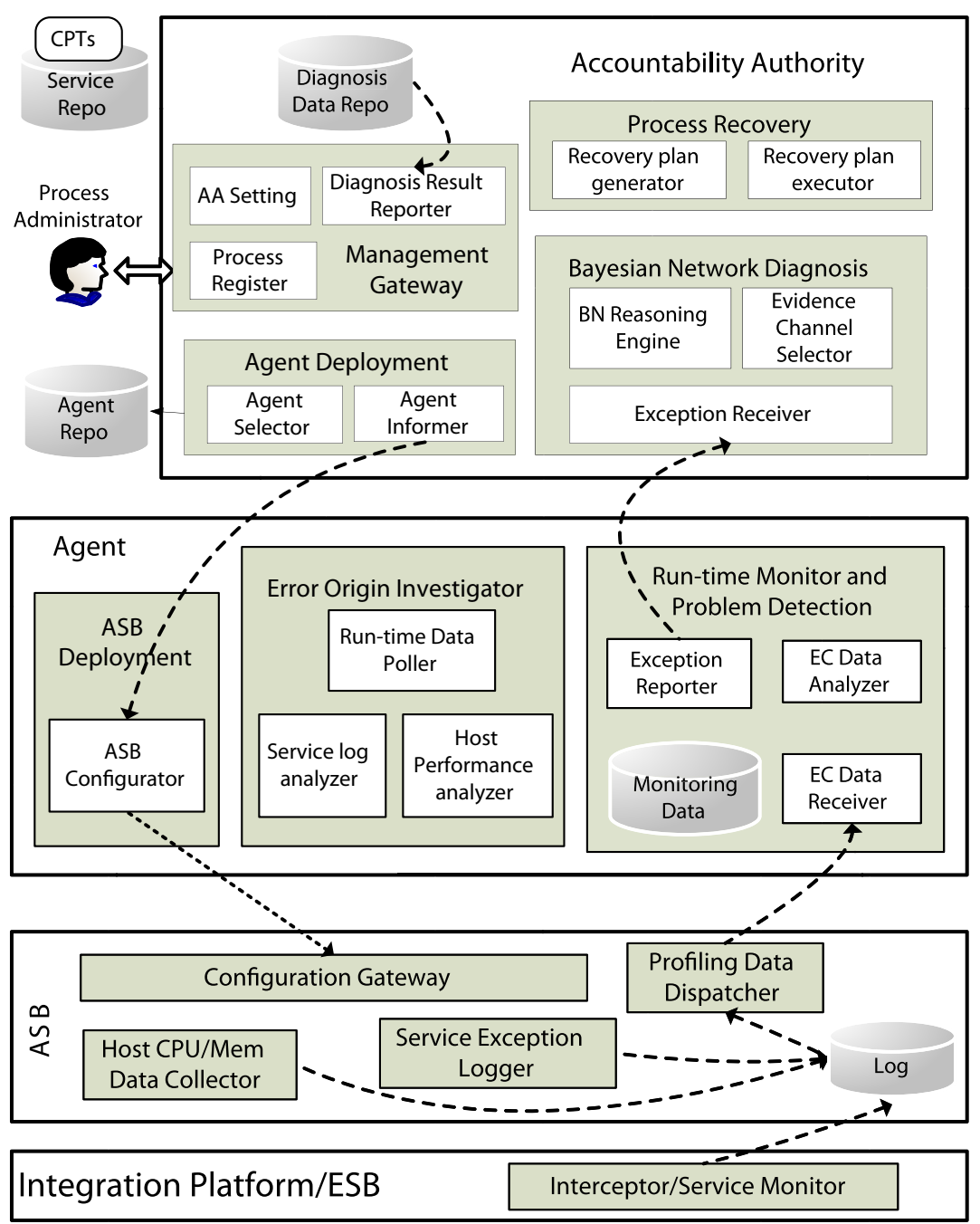

Fig. 3. LLAMA accountability architecture and components

\subsection{LLAMA Components}

In this section, we focus our discussion on the LLAMA accountability core (Fig. 3), comprised of the ASB, Agents, and the Accountability Authority.

Accountability Authority (AA). AA is the LLAMA component that performs intelligent management for the deployment, diagnosis, and reconfiguration of a service process. AA has the following responsibilities: (1) receive process 
management requests from process administrators; (2) perform initial deployment and configuration of the accountability framework once the process is submitted (in BPELQ) for management; (3) perform root-cause diagnosis when exceptions are reported (sometimes concurrently) from Agents; (4) perform an automated process reconfiguration to recover process execution. The primary components of AA (in Fig. [3) are as follows.

- The Management Gateway, which serves as the portal for process administrators, allows them to submit processes and QoS constraints for accountability management. It also allows administrators to configure AA settings, such as the Evidence Channel selection strategy [4]. Moreover, it reports diagnosis results saved in the diagnosis data repository to administrators.

- At the deployment phase, the Bayesian Diagnosis Engine performs Evidence Channel selection algorithms [6] to decide the most cost-effective service data collection locations. At run-time, it performs Bayesian Network reasoning to identify the most suspicious services that may be the rootcause of problems. It also works with Agents to confirm the root cause and investigate possible error sources (e.g., service logic, host, network, etc.).

- The Agent Selector decides the set of Agents with the least cost to cover all services using good algorithms, such as the greedy set covering algorithm in [4.

- The Agent Informer notifies Agents about their selected Evidence Channels, i.e. the services each Agent is responsible for monitoring; the type of data to be reported; the frequency of data collection; and the criteria to decide if a service is normal or has an exception.

- The Exception Receiver receives filtered exception data from Agents, which usually triggers diagnosis procedures.

- The Process Recovery component generates process reconfiguration plans and executes them by notifying the ASB, via Agents, to reroute the process path using new services.

Accountability Agents. Agents are the intermediaries between where data is collected (i.e., ASB) and where it must be sent for analysis and diagnosis (i.e., AA). They are responsible for the following tasks: (1) configure evidence channels on ASB; (2) perform run-time data analysis based on the information pushed by ASB; (3) report exceptions to AA; and (4) initiate error origin investigation upon the request of AA. The components of Agents as illustrated in Figure 3 include:

- The ASB Configurator receives instructions from AA about which services are selected as Evidence Channels. Agents in turn contact ASB where a service is hosted and instructs ASB to send all relevant data about the service to the Agent.

- The EC Data Receiver and Analyzer receives pushed data from Evidence Channels located on ASB. It then applies exception criteria sent by AA on the data. Any data that meet the exception criteria are forwarded to Exception Reporter. 
- The Exception Reporter promptly reports any abnormal situations detected by EC Data Analyzer to AA, which initiates the diagnosis process.

- The Run-time Data Poller assists AA to confirm root cause locations during the diagnosis process. It sends requests to ASB for data not in Evidence Channels, filters those data according to the exception criteria and sends them to AA.

- The Error Origin Investigator requests further information from ASB to determine if the source of an error is due to network, host, or the service itself once a problematic service is identified by AA.

LLAMA ASB . LLAMA ASB extends normal ESB capabilities by providing an API and framework for Agents to collect service performance data. Data can be pushed or pulled, and collected and sent at configurable intervals. LLAMA ASB can be installed on any existing ESB framework as long as it supports service request interception or other means of collecting service data. The following are the features of the LLAMA ASB (Fig. 3) that give it the unique monitoring capabilities.

- The Configuration Gateway (CGW) provides the service interface for configuring internal LLAMA ASB capabilities by Agents. CGW is used to configure service and host profiling data collection, including data collection and dispatch frequencies. Moreover, it allows the selection of Evidence Channels and dynamic routing configuration.

- Profiling Interceptors intercept service request/response messages and collect both timestamp and message output data. This data is stored in the ASB $\log$.

- Communication Interceptors give the LLAMA framework its reconfiguration capabilities by providing the underlying mechanisms to route, forward, and redirect service requests [13] via the configuration of the routing table located on the ASB. It can accommodate both centralized and distributed process control models.

- The Service Logger collects software exceptions generated from service execution and stores them in the log. The exception log may be used for detailed diagnosis to understand the service failure.

- The Host Data Collector collects detailed statistics about the host's performance. ASB collects CPU, memory, and network data for each host on which it is deployed. The host data collector can be configured to collect data at intervals specified via CGW.

- The Data Dispatcher allows ASB to manage how data is pushed to Agents and can be configured to send data at intervals specified via CGW.

\subsection{Error Origin Investigation}

The LLAMA framework is unique in being able to diagnose process problems and heal the process if necessary by triggering a reconfiguration. It is important to pinpoint the exact cause of a process problem since it will influence the recovery 
process. For example, if the network is at fault, a replacement service must be located outside of the original service's network. Similar action is taken if the host is at fault.

When an end-to-end QoS violation is detected during process execution, a list of likely causes is generated from the AA Bayesian network diagnosis engine. AA asks Agents to query data from ASB about a service, and such data is compared with the specified criteria to determine if an instance of data is an exception. All exceptions are gathered by Agents and sent to AA. AA then needs to determine the root source of the error. For this the Agent's error origin investigator is invoked by AA and the Agent requests further information from ASB on which the problematic service is deployed to determine the source of error. The Agent performs this investigation by looking up both system and service information, collected continuously by the ASB's Host Data Collector, based on the time at which the problematic service execution occurred. The Agent attempts to identify error causes by examining the sources listed below, based on some preliminary metrics.

- Service exceptions: Exceptions may be raised within a service's own implementation and may not only affect the correctness of a service's execution, but also could effect its performance. Such exceptions are recorded and stored within the ASB log. During error origin investigation, the log is checked to determine if any exceptions had been raised during the execution of the service.

- CPU utilization: High CPU utilization on a host will cause delays in all executing services. ASB by default collects CPU utilization data at 5 second intervals (using the top Linux command) and records them in a log. For analyzing CPU utilization, we refer to current research in load balancing literature to arrive at a problematic load threshold of $95 \%$ 14. We compare this figure with the average CPU utilization over the time when the service was executed.

- Memory consumption: When memory consumption is high relative to available physical memory, paging may occur thus reducing system performance. ASB records memory consumption as a percentage of total available physical memory at regular intervals (using the free Linux command). If physical memory utilization exceeds $100 \%$ then we may assume that memory consumption is a contributing factor to the service's poor performance.

- Network and infrastructure latency: ASB records timestamps at both the beginning and the end of service executions. Using this data, Agents can calculate the time taken between the end of one service execution, and the beginning of the execution of the next service in the process. This time represents a combination of network time, as well as time spent in the communication infrastructure on each respective host. Agents can leverage network traffic data associated with each host (also collected by the ASB using the ntop Linux command) to further narrow the problem source to that of the network or host infrastructure. We apply techniques discussed in 15 to derive thresholds to which we compare network data when error origin investigation is invoked. 
Although Agents may inspect all of the above data sources, sometimes it is still very difficult to distinguish between the various possible sources of service performance problems. In other words, the results from Agent investigations are by no means completely reliable. Such investigations are based on artificial thresholds and patterns that are empirically estimated and may vary from system to system. On the other hand, the investigation reliability may increasingly improve as more historical data is collected and correctly analyzed.

Once error investigation has been concluded by Agents, and the likely root cause of a process error is reported, AA then makes a reconfiguration decision. Various techniques for service selection and process reconfiguration [11, as well as ASB support for reconfiguration 13 , has been presented in our earlier reports.

\section{Empirical Results}

A prototype of LLAMA has been implemented by building the ASB as an extension to the Mule ESB, and constructing the AA and Agents. We now study the overhead of the LLAMA prototype and the accuracy of its diagnosis process.

\subsection{Example Scenario: Print and Mail}

We use an example BP to test the effectiveness of the LLAMA framework. It is a $\mathrm{BP}$ for targeted mass mail advertising referred to as the Print and Mail process (Figure 4). For example, with such a BP a plumber can send fliers to all addresses in a certain zip code where the houses are older than 20 years.

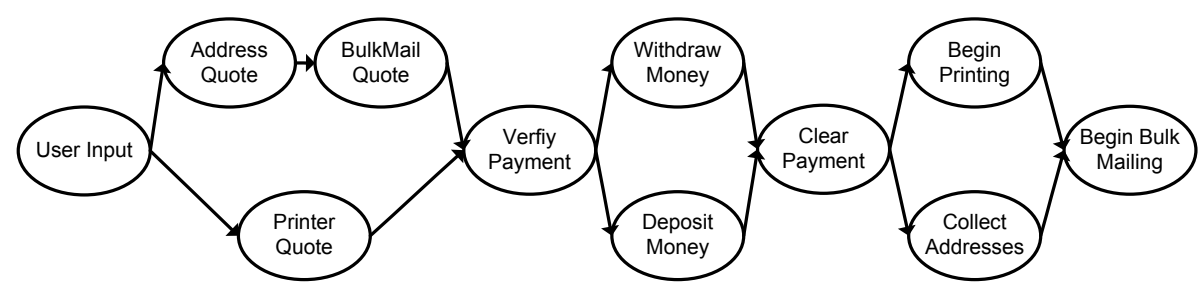

Fig. 4. The Print and Mail Business Process

The process begins with the user's specifications, including address specifications and the image to provide to the printer. Next, various pricing quotes are gathered from print vendors, address providers, and bulk mailers. The process may retrieve quotes from several of these services in parallel to find the best one. Once the quotes have been settled, the form of payment is verified and the payments is made. In final stage, the bulk mail process can begin once the addresses are collected from the address vendor and the printing process has been completed. 


\subsection{Monitoring Overhead}

Monitoring and problem diagnosis can potentially create overhead in any executing software system. In this section, we measure the overhead of the LLAMA framework for the Print and Mail BP. For this experiment, we deploy all services and two Agents on a single host. Therefore, data is being collected about all services. Moreover, six of the services are selected as Evidence Channels so that data from these services are being continuously pushed to the Agents. We set up three test scenarios: 1) execute the process 50 times with all profiling interceptors turned off, ensuring that there is no data collection overhead, 2) execute the process 50 times with all profiling interceptors activated ensuring that all data collection is taking place, and 3) the same as scenario 2, except that 6 Evidence Channels are configured and are pushing data at 5 second intervals to the Agents. Results from three separate runs are depicted in Table 1 . These results indicate that there is no statistically significant difference between the first two scenarios and therefore monitoring overhead in negligible. Pushing data to local Agents, however, can create from 1\% to 7\% additional overhead.

Table 1. Monitoring Overhead (average of 50 runs for each test)

\begin{tabular}{|l|c|c|c|}
\hline & Test 1 Average & Test 2 Average & Test 3 Average \\
\hline No monitoring & $29033 \mathrm{~ms}$ & $27922 \mathrm{~ms}$ & $28323 \mathrm{~ms}$ \\
\hline Data collection at ASB & $29053 \mathrm{~ms}$ & $28515 \mathrm{~ms}$ & $27862 \mathrm{~ms}$ \\
\hline Data delivered to Agents & $29354 \mathrm{~ms}$ & $29199 \mathrm{~ms}$ & $30437 \mathrm{~ms}$ \\
\hline
\end{tabular}

\subsection{LLAMA Diagnosis Accuracy}

In this section, we study how accurately LLAMA can find root causes when a $\mathrm{BP}$ is reported to have unacceptable performance according to specified requirements. The detailed design of the diagnosis engine and parameter setup have been reported in [4. Here we test the actual performance in a deployed system.

To simulate performance problems, we inject 50-second delays into certain services in the process following the pre-defined reputation of those services. For example, if service A's reputation is 0.9 , then there is 0.1 probability of delay injection. Since the response time of every service in our test is set to 10 seconds or less, we choose the acceptable response time for each service to be 15 seconds.

We have tested the Bayesian network reasoning as shown in Table 2, We find that if we inject delay to only one service, the Bayesian network reasoning can always find the correct root cause in 2 diagnosis rounds. If we inject delay in two services, the Bayesian engine can find out at least one root cause in 4 rounds.

We test two probability thresholds, 0.1 and 0.3 , for initiating service inspection after the diagnosis report on the probability of service error. Obviously, a lower threshold will trigger more diagnosis rounds and cause a longer diagnosis duration. For single-cause cases, we find the threshold of 0.3 is good enough. But for multi-cause cases, the low threshold of 0.1 can help detect more problematic 
Table 2. Diagnosis Performances with Thresholds of 0.1 and 0.3

\begin{tabular}{|c||c|c|c|c|c|c|c|c|c|c|}
\hline TH=0.1 case & 1 & 2 & 3 & 4 & 5 & 6 & 7 & 8 & 9 & 10 \\
\hline BP duration & $1 \mathrm{~m} 32 \mathrm{~s}$ & $1 \mathrm{~m} 31 \mathrm{~s}$ & $1 \mathrm{~m} 40 \mathrm{~s}$ & $1 \mathrm{~m} 40 \mathrm{~s}$ & $2 \mathrm{~m} 12 \mathrm{~s}$ & $1 \mathrm{~m} 58 \mathrm{~s}$ & $1 \mathrm{~m} 33 \mathrm{~s}$ & $2 \mathrm{~m} 6 \mathrm{~s}$ & $2 \mathrm{~m} 6 \mathrm{~s}$ & $2 \mathrm{~m} 7 \mathrm{~s}$ \\
\hline Diag. duration & $1 \mathrm{~s}$ & $1 \mathrm{~s}$ & $2 \mathrm{~s}$ & $1 \mathrm{~s}$ & $2 \mathrm{~s}$ & $3 \mathrm{~s}$ & $1 \mathrm{~s}$ & $2 \mathrm{~s}$ & $2 \mathrm{~s}$ & $2 \mathrm{~s}$ \\
\hline \#Diag round & 7 & 4 & 4 & 2 & 6 & 6 & 6 & 4 & 8 & 7 \\
\hline Detection ratio & $1 / 1$ & $1 / 1$ & $1 / 1$ & $1 / 1$ & $1 / 2$ & $2 / 2$ & $2 / 2$ & $1 / 2$ & $3 / 4$ & $2 / 4$ \\
\hline \hline TH=0.3 cases & 1 & 2 & 3 & 4 & 5 & 6 & 7 & 8 & 9 & 10 \\
\hline BP duration & $1 \mathrm{~m} 33 \mathrm{~s}$ & $1 \mathrm{~m} 32 \mathrm{~s}$ & $1 \mathrm{~m} 45 \mathrm{~s}$ & $1 \mathrm{~m} 38 \mathrm{~s}$ & $2 \mathrm{~m} 5 \mathrm{~s}$ & $1 \mathrm{~m} 40 \mathrm{~s}$ & $1 \mathrm{~m} 36 \mathrm{~s}$ & $2 \mathrm{~m} 6 \mathrm{~s}$ & $2 \mathrm{~m} 8 \mathrm{~s}$ & $2 \mathrm{~m} 6 \mathrm{~s}$ \\
\hline Diag. duration & $1 \mathrm{~s}$ & $1 \mathrm{~s}$ & $1 \mathrm{~s}$ & $1 \mathrm{~s}$ & $2 \mathrm{~s}$ & $2 \mathrm{~s}$ & $1 \mathrm{~s}$ & $2 \mathrm{~s}$ & $2 \mathrm{~s}$ & $2 \mathrm{~s}$ \\
\hline \#Diag round & 3 & 2 & 3 & 2 & 3 & 2 & 2 & 4 & 3 & 3 \\
\hline Detection ratio & $1 / 1$ & $1 / 1$ & $1 / 1$ & $1 / 1$ & $1 / 2$ & $1 / 2$ & $1 / 2$ & $1 / 2$ & $1 / 4$ & $1 / 4$ \\
\hline
\end{tabular}

services. The system may not catch all problematic services in multi-cause cases since if we inject errors in two services that are on the same path of a process, only the first one (executed first) may be reported as the root cause. We will continue to improve the AA's diagnosis capability in our future work.

\section{Related Work}

Most enterprise software vendors have produced ESB products, including IBM, BEA, Sonic, Iona, Oracle, Cape Clear, etc. Several open source ESB projects have also been implemented, including Celtix, ServiceMix, Apache Synapse, Open ESB, etc. To our knowledge, none have included the sophisticated diagnosis capability like in our project. Our current implementation of LLAMA has been designed to run atop the Mule ESB [9], because it is a stable open-source project with highly extensible features. Mule allows customized interception and message transformation mechanisms to support profiling collection and process reconfiguration. However, LLAMA can run on any ESB which supports some means of collecting service invocation data.

In [16], Errandi et. al. present wsBus, a Web service middleware for reliable and fault-tolerant computing. To handle faults, wsBus performs run-time monitoring of functional and QoS aspects, as well as run-time adaptation based on the policies specified by users. However, the monitoring and adaptation components of wsBus are designed and executed on an individual service basis, whereas the LLAMA framework uses the accountability model to monitor the end-to-end quality of processes and adapt at the process level with an alternate path. In addition, the configuration gateway and rerouting facility in the LLAMA ESB are designed to be transparent and very efficient.

In [15], a performance metrics monitoring and analysis middleware named Tiresias is developed to collect application level (e.g., response time) and systemlevel performance metrics (e.g., CPU usage, memory usage, and network traffic). These performance data are used to make black-box failure-prediction through trend analysis. Our LLAMA framework differs from Tiresias in terms of data 
dependency reasoning and analysis since our target applications are business processes.

Autonomic computing 17 aims to develop computing systems that can manage themselves given high-level objectives from administrators. The essence of autonomic computing systems is self-management, which comprises four aspects: self-configuration, self-optimization, self-healing, and self-protection. Accountability provides the solid foundation for the fully-scaled autonomic computing. It targets the self-healing and self-optimization aspects of autonomic computing - a system automatically detects, diagnoses, and repairs software and hardware problems. They share the common goal of identifying, tracing, and determining the root cause of failures in complex computing systems.

The goal of fault tolerance is to enable systems to continually operate properly. Therefore, the major technologies to achieve fault tolerance focus on recovery from failures. Those technologies mainly include replication, checkpointing and recovery lines [18. Accountability complements dependable SOA systems by being able to identify and determine problematic services (e.g., violations of SLAs) and then defuse the problem.

\section{Conclusion}

As enterprises continue to construct a larger proportion of their business processes from outsourced third-party services, there will be an increased need to maintain the run-time knowledge of these services in order to accurately diagnose difficultto-pinpoint problems when they occur. To address this emerging trend, we have developed the LLAMA middleware framework. It includes components to help monitor services on behalf of a process user, find the root cause of problems when they occur, and perform reconfigurations if necessary.

Due to space constraints, we present only the overall architecture, while omitting many algorithmic foundations and system details, which may be found in our earlier reports [46]. From the experiments reported in this paper, we have discovered that the LLAMA middleware creates little overhead, and provides a reasonably accurate root cause diagnosis. We believe that this is a promising approach toward implementing accountable SOA systems.

\section{References}

1. Papazoglou, M.P., Traverso, P., Dustdar, S., Leymann, F.: Service-oriented computing: State of the art and research challenges. IEEE Computer 40, 38-45 (2007)

2. Bichler, M., Lin, K.J.: Service-oriented computing. IEEE Computer 39(3), 99-101 (2006)

3. Huhns, M.N., Singh, M.P.: Service-oriented computing: Key concepts and principles. IEEE Internet Computing (January-February 2005)

4. Zhang, Y., Lin, K.J., Hsu, J.Y.: Accountability monitoring and reasoning in serviceoriented architectures. Journal of Service-Oriented Computing and Applications (SOCA) 1(1) (2007) 
5. Korb, K.B., Nicholson, A.E.: Bayesian Artificial Intelligence. Chapman \& Hall/CRC, London (2004)

6. Zhang, Y., Panahi, M., Lin, K.J.: Service process composition with QoS and monitoring agent cost parameters. In: IEEE Joint Conf. on E-Commerce Technology (CEC 2008) and Enterprise Computing (EEE 2008) (July 2008)

7. Chappell, D.: Enterprise Service Bus. O'Reilly Media, Sebastopol (2004)

8. CapeClear: Capeclear bam (2008), http://developer.capeclear.com/

9. MuleSource: Mule 2.0 (2007), http://mule.codehaus.org/display/MULE/Home

10. Oasis: WSDM (2008), http://www.oasis-open.org/

11. Yu, T., Lin, K.J.: Service selection algorithms for composing complex services with end-to-end QoS constraints. In: Benatallah, B., Casati, F., Traverso, P. (eds.) ICSOC 2005. LNCS, vol. 3826, pp. 130-143. Springer, Heidelberg (2005)

12. Yu, T., Zhang, Y., Lin, K.J.: Efficient algorithms for web services selection with end-to-end qos constraints. ACM Transactions on the Web (May 2007)

13. Lin, K.J., Panahi, M., Zhang, Y.: The design of an intelligent accountability architecture. ICEBE, 157-164 (2007)

14. Balasubramanian, J., Schmidt, D.C., Dowdy, L., Othman, O.: Evaluating the performance of middleware load balancing strategies. In: EDOC 2004: Proceedings of the Enterprise Distributed Object Computing Conference, Eighth IEEE International, pp. 135-146. IEEE Computer Society, Washington (2004)

15. Williams, A.W., Pertet, S.M., Narasimhan, P.: Tiresias: Black-box failure prediction in distributed systems. In: The 15th International Workshop on Parallel and Distributed Real-time Systems. IEEE, Los Alamitos (2007)

16. Erradi, A., Maheshwari, P., Tosic, V.: Policy-driven middleware for self-adaptation of web services compositions. In: Middleware, pp. 62-80 (2006)

17. Kephart, J.O., Chess, D.M.: The vision of autonomic computing. IEEE Computer 36(1), 41-50 (2003)

18. Tsai, W.T., Song, W., Paul, R., Cao, Z., Huang, H.: Services-oriented dynamic reconfiguration framework for dependable distributed computing. In: Proceedings of the 28th Annual International Computer Software and Applications Conference (COMPSAC 2004), pp. 554-559 (2004) 